\title{
Generating Adaptive Presentations of Hydrologic Behavior
}

\author{
Martin Molina and Victor Flores \\ Department of Artificial Intelligence, Universidad Politécnica de Madrid, \\ Campus de Montegancedo S/N 28660 Boadilla del Monte, Madrid, Spain. \\ \{mmolina,vflores\}@fi.upm.es
}

\begin{abstract}
This paper describes a knowledge-based approach for summarizing and presenting the behavior of hydrologic networks. This approach has been designed for visualizing data from sensors and simulations in the context of emergencies caused by floods. It follows a solution for event summarization that exploits physical properties of the dynamic system to automatically generate summaries of relevant data. The summarized information is presented using different modes such as text, 2D graphics and 3D animations on virtual terrains. The presentation is automatically generated using a hierarchical planner with abstract presentation fragments corresponding to discourse patterns, taking into account the characteristics of the user who receives the information and constraints imposed by the communication devices (mobile phone, computer, fax, etc.). An application following this approach has been developed for a national hydrologic information infrastructure of Spain.
\end{abstract}

\section{Introduction}

One of the main tasks of water control centers is to help to react in the presence emergency situations as a consequence of floods. In this context, the state of the hydrological basin is measured with the help of sensors that send periodically to the control center quantitative values about rainfall, water levels and flows. When the dimension and complexity of the information is high, a large number of values need to be summarized and adequately presented. Automatic tools can help in this task in order to minimize the time response of human operators in the presence of emergencies. In general, this is a complex task that normally requires specific knowledge about the dynamic system. In fact, summarization and presentation modeling have received important attention in the research community of Artificial Intelligence where certain general solutions have been proposed [1][2].

In this paper we describe an innovative knowledge-based approach to perform automatically this task in the context of the surveillance of hydrologic networks. The main contribution of our approach is that we take the advantage of the system representation to support specific strategies for summarizing and presentation. As a result, we propose an efficient solution to generate automatically adaptive multimodal reports according to prefixed management goals. In the following, the paper describes a general view of the method as a knowledge-based architecture. It is illustrated with the application in the field of hydrology. At the end of the paper we make a comparative discussion with similar approaches.

In "Intelligent data Engineering and Automated Learning IDEAL 2006”, E. Corchado, H. Yin, V. Botti, C. Fyfe (Eds.). 7th International Conference, IDEAL 2006, LNCS 4224, pp. 896 - 903. Burgos, Spain, September 2006. C Springer-Verlag Berlin Heidelberg 2006. 


\section{A General View of the Method}

The goal of the method is to present relevant information about the behavior of the dynamic system at an adequate level of abstraction close to the decision processes. This information is presented as concise as possible and it is accompanied with additional information to facilitate a complete understanding. The information is presented in different modes (text, graphics, animations, etc.) using different devices for reception (computer, mobile phone, fax, etc.).

The method has been designed following a knowledge-based approach (according to recent knowledge engineering methodologies [3]) with a set of general inference steps that use domain specific knowledge. The method performs two main tasks: (1) summarize the most important information (i.e., what to inform) and (2) generate a presentation plan according to the type of end-user and the communication media (how to present the information).

\subsection{Summarization}

Our method for summarization is based on an explicit representation of the dynamic system (see [4] for more details). The method was designed to simulate professional human operators in control centers with partial and approximated knowledge about the dynamic system. This is formulated following an approach based on representations and ontologies of qualitative physics [5] [6] [7].

The structure of the dynamic system is represented with hierarchies of components with quantities for physical magnitudes and qualitative states. The model includes a simplified view of the system behavior represented with causal relations between quantities with labels such as temporal references about approximated delay and type of influence. Historical values also help to represent information about behavior (e.g., average values, maximum historical values, etc.). This representation includes a qualitative interpretation model to determine the qualitative state of every single component from physical quantities and an aggregation model to determine the state of complex components based on the state of simpler components (both models are represented using rules).

The representation also includes what we call the salience model to determine when certain event is relevant and should be reported to the operator. In general, we consider a relevant event as an event that (1) changes with respect to the immediate past and (2) produces a change in the distance between the state of the dynamic system and the desired state established by the management goals. This distance is a way to quantify the degree of relevance of events. Based on our assumption for system modeling (with approximated knowledge for system behavior) the representation of relevance establishes preferences between states based on relations that summarize sets of behaviors [4].

The strategy of inference receives as input sensor data and uses this representation to produce what we call a summarization tree. This tree is a particular representation that summarizes the most relevant information to be reported to the user. To construct this tree, the inference procedure performs the following steps. For every single component, its qualitative state is computed using as input the interpretation model and the quantitative measures of sensors. Then, these states are ranked according to 
the heuristics of the salience model. Causes and effects are removed following the order in this set. Finally, the states of similar components are condensed by aggregation (states of components with the same type are aggregated by the state of a more global component) and abstraction (two states of components of different type are abstracted by the most relevant state). This produces the summarization tree (see figure 2) where the root is the most representative event and the branches include aggregated and abstracted states. The salience model plays the role of control knowledge in the inference procedure because it directs the search for qualitative states of complex components using first the most relevant states of simpler components. This strategy provides an adequate condensation of information by aggregation based on relevance.

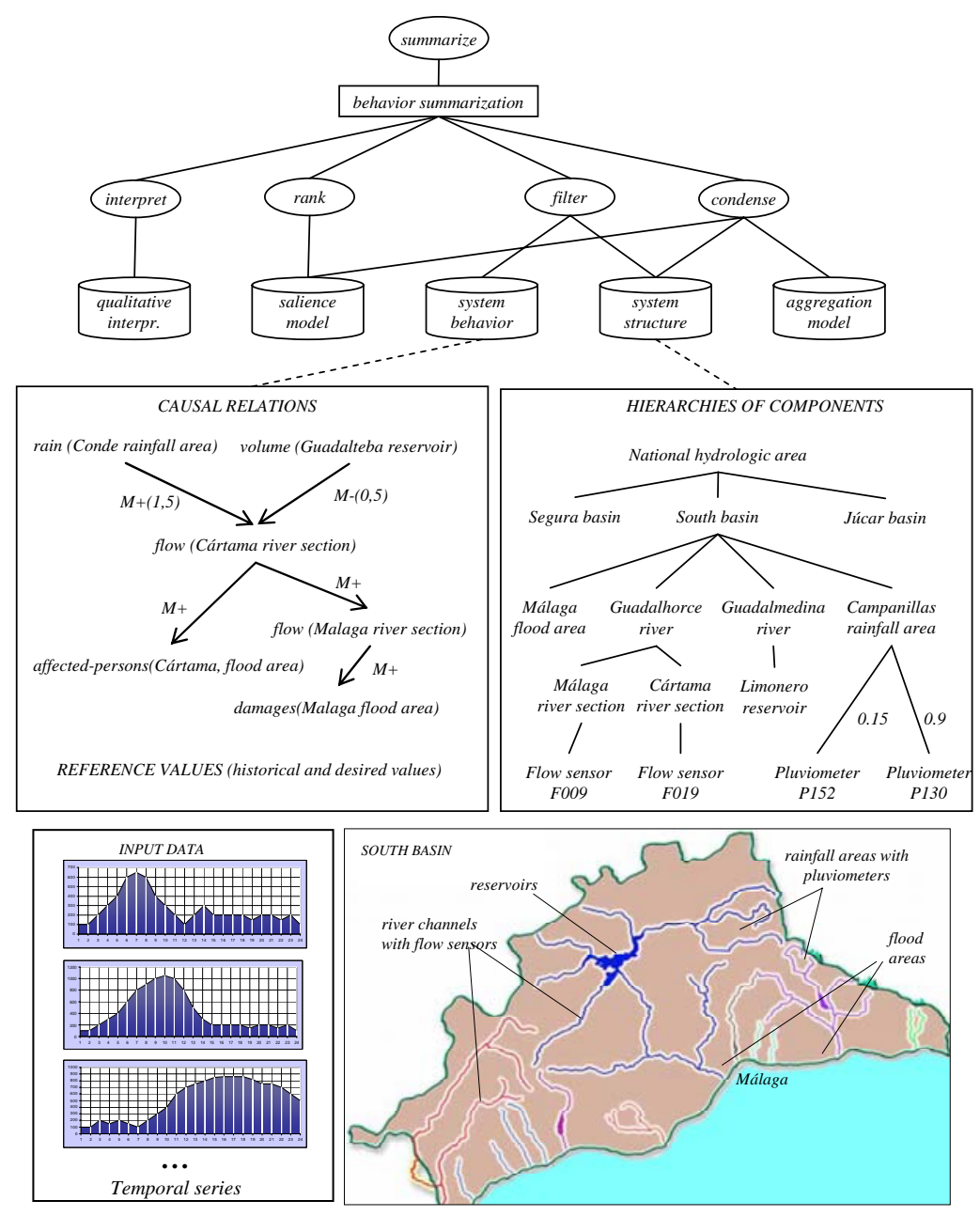

Figure 1: A global view of the method for summarization in the hydrologic domain. 


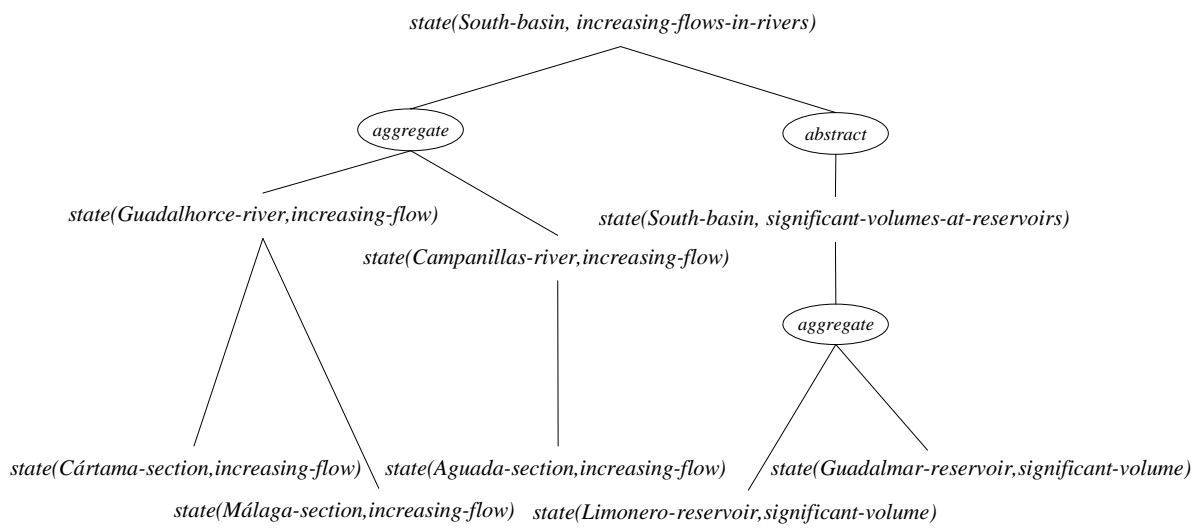

Figure 2: Example of summarization tree.

\subsection{Presentation Planning}

Once the relevant information to be reported to the operator is determined (what to inform) it is necessary to generate an adequate presentation plan (how to present the information). For this purpose, the method gathers related information that helps to understand relevant events and determines the type and detail of the presentation taking into account the end-user and the communication devices (mobile telephone, fax, computer screen with 3D animations, etc.).

In order to bring together the information related to relevant events, the method develops discourse strategies that connect information modules with rhetorical relations (as used in Rhetorical Structure Theory [8]). For this purpose, we have formulated a set of model-based gathering strategies for rhetorical relations applicable in our context of dynamic system surveillance (see table 1) following the system representation and our notion of relevance.

\begin{tabular}{|c|l|}
\hline $\begin{array}{c}\text { Rhetorical } \\
\text { relation }\end{array}$ & \multicolumn{1}{c|}{ Gathering strategy } \\
\hline causes & $\begin{array}{l}\text { The method gathers related causes. A cause is related if its state has changed in } \\
\text { the last } \Delta t \text {, where } \Delta t \text { is the delay between each cause and the event. }\end{array}$ \\
\hline effects & $\begin{array}{l}\text { The method gathers related effects. An effect is related if its state is not assumed } \\
\text { by default. Default values are either goal values or values already reported to the } \\
\text { user. }\end{array}$ \\
\hline contrast & $\begin{array}{l}\text { The method gathers the goal value of an event according to the management } \\
\text { strategy. This goal value is informed in contrasts to the current value of the event. }\end{array}$ \\
\hline evidence & $\begin{array}{l}\text { The method gives evidence of a fact with the measured values of the } \\
\text { corresponding sensor. }\end{array}$ \\
\hline details & $\begin{array}{l}\text { Details of the state of an aggregated component correspond to the state of the } \\
\text { simpler component with greater distance to the goal value and a list of the rest of } \\
\text { components with the same state. }\end{array}$ \\
\hline
\end{tabular}

Table 1: Examples of gathering strategies for rhetorical relations.

To articulate rhetorical relations for a particular discourse the method uses discourse patterns. Each pattern is a template that expresses how to develop a part of the discourse. The total set of discourse patterns is implemented as the knowledge base of a planner. Here, we follow the terminology of HTN planning (Hierarchical 
Task Network) [9] with planning-tasks, planning-methods, preconditions and operators. In our method, each planning-task corresponds to a communication goal (e.g., inform about the details of a relevant event) and each planning-method corresponds to a discourse pattern. A pattern has a set of communication sub-goals that corresponds to the rhetorical relations. The preconditions formulate the applicability conditions of the pattern with the following issues: (1) what, i.e., the characteristics of the event to be informed, (2) who, i.e., the type of user who receives the information (level of background knowledge, available time, etc.), (3) where, i.e., presentation constraints imposed by the communication device (mobile phone, fax, web page, etc.). The preconditions of patterns invoke specialized functions (e.g, causes $(x, y)$ or effects $(x, y))$ to gather additional information according the rhetorical relations. The knowledge base of the planner also includes basic operators for atomic communication goals, i.e., for goals that are not divided into other sub-goals. These operators are implemented as specialized presentation primitives in the form of textual descriptions and parameterized functions that compose text, illustrate with 2D graphics and construct 3D animations.

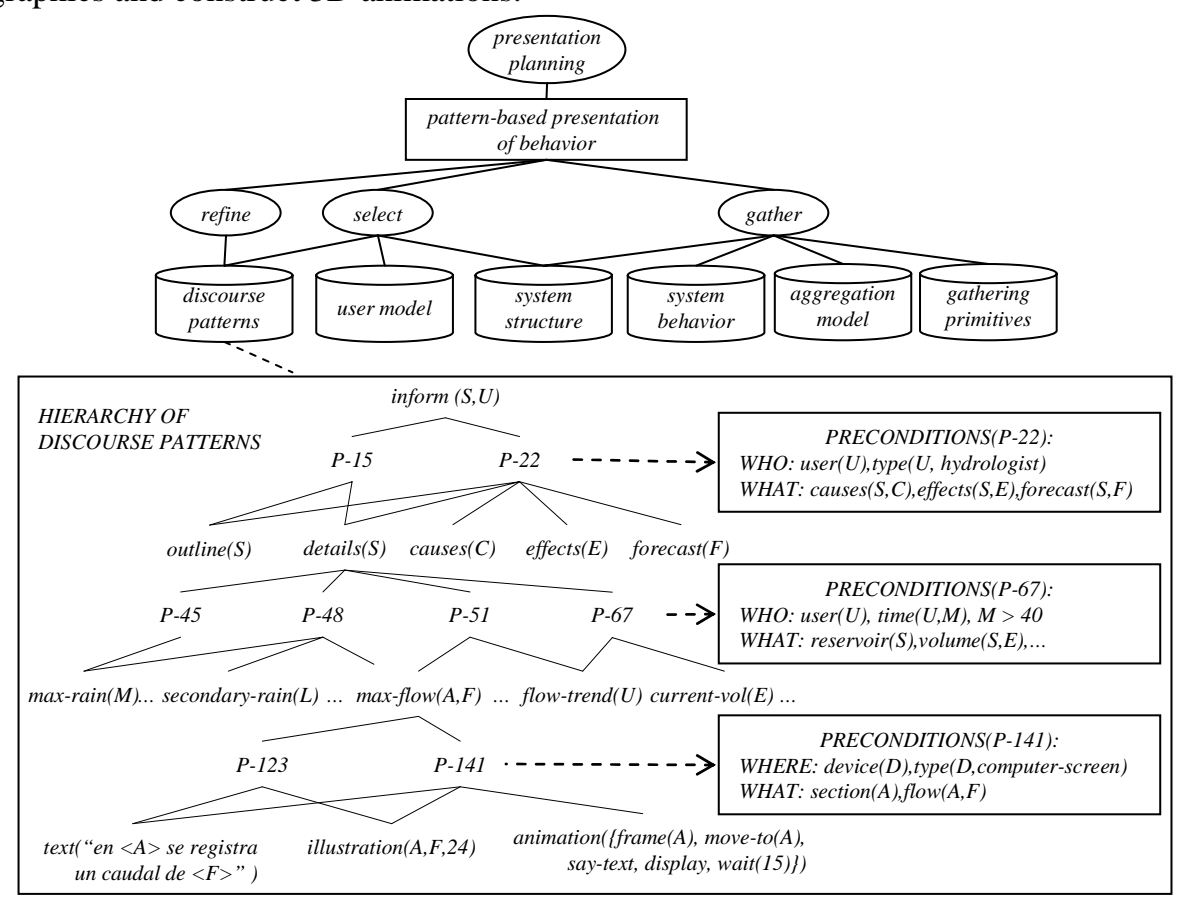

Figure 3: Summary of the method for presentation planning.

Figure 3 shows a summary of the method for presentation planning. To construct the complete plan the method follows a recursive planning procedure that, in every step performs the following subtasks: (1) select a discourse pattern that satisfies the preconditions, (2) gather additional information according to the preconditions, and (3) refine the communication goal with the sub-goals. The second subtask uses the system model (structure and behavior) together with the aggregation model to construct additional summarization trees for the presentation. 


\section{Discussion}

The previous method has been developed to work with real time data of SAIH systems (SAIH: Spanish acronym for Automatic Information System in Hydrology) developed by a National Programme of Spain. It was conceived to help to react in the presence emergency situations as a consequence of river floods, as a component of a more complex intelligent system for emergency management [10]. In this context, information is received periodically in a control center about rainfall at certain locations, water levels and flow discharge of reservoirs and flows in certain river channels. Figure 4 shows an example of the presentation that can be generated with the help of the method presented in this paper.

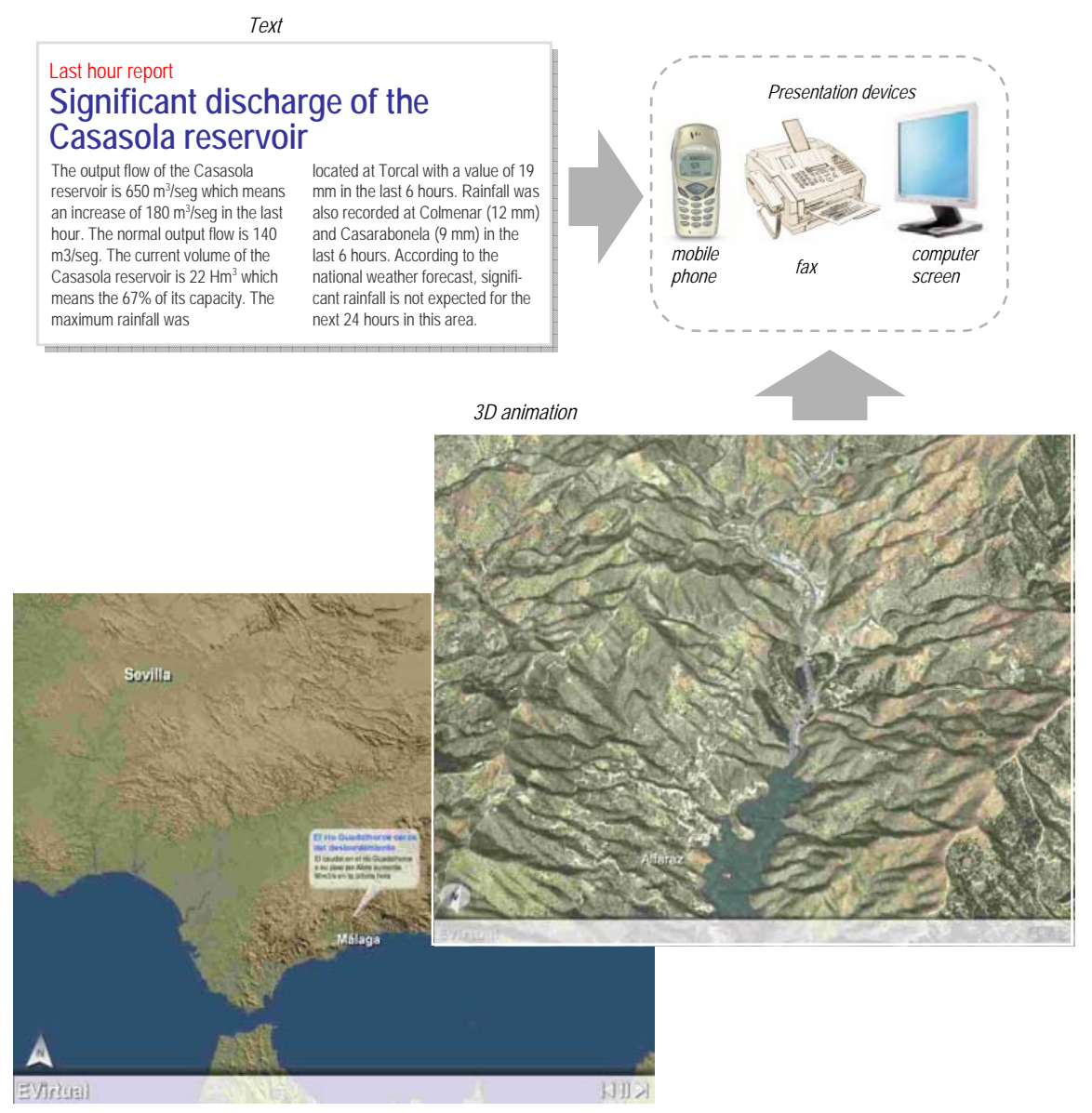

Figure 4: Examples of information reported in the field of hydrology. 
Our method for presentation can be compared to techniques of artificial intelligence such as explanation generators and presentation modeling. For example, our method generates explanations that help to understand the relevant events. Compared to methods for diagnosis [11] our approach does not look for hidden causes for a set of given symptoms. Instead, it complements the relevant information by selecting data in the measured information using causal knowledge.

Compared to the field of presentation models we integrate and extend some general ideas about multimodal presentation planning of the WIP project [2] [12] for the case of dynamic systems (for example, our method defines gathering primitives specialized in the system model). For the particular case of text composition we follow a template-based approach [13]. Concerning text composition, our solution also presents certain similarities for the case of ILEX [14] although with different model representation, notion of relevance and abstraction method.

Related to our method, other solutions for presentation generation in the field of meteorology have been proposed. For example, the system of Kerpedjiev [15] follows a multimodal approach based on WIP [12] but it is restricted to documents and 2D graphics. In this field there are other examples for presentation specialized in natural language generation [16][17].

\section{Conclusions}

In summary, the paper presents a knowledge-based approach to generate adaptive presentations in the field of hydrology. The method has been designed to work in the context of the surveillance of the behavior of a hydrological basin. Our approach contributes with the architecture of a method that identifies different types of knowledge about the dynamic system (i.e. a hydrological basin) and model-based strategies for presentation planning that are organized according to rhetorical relations for discourse generation. The approach is, up to our knowledge, an innovative application in the hydrologic domain.

The method has been designed to operate with data from a national hydrologic information infrastructure (the SAIH systems in Spain). A preliminary validation of the method in this context with partial models has been carried out with satisfactory results. Currently, we are working on a more extensive evaluation with complete models. The presentation method was developed for the domain of hydrology, but we are also working on a general approach to be used in other domains.

Acknowledgements. This research work has been supported partially by the Ministry of Education and Science of Spain within the E-VIRTUAL project (REN2003-09021C03-02). The Ministry of Environment of Spain (Dirección General del Agua) provided information about the domain in hydrology. The authors would like to thank Sandra Lima, M. Carmen Martín, Nuria Andrés and Enrique Parodi for their work on the implementation of the presentation method. 


\section{References}

1. Wahlster W.: "User and discourse models for multimodal communication". In Sullivan, J. W., Tyler, S. W. \& Sherman, W. editors, Intelligent User Interfaces. New York: ACM Press, S. 45-67. 1991.

2. Maybury M. T.: "Generating Summaries from Event Data". Information Processing and Management: an International Journal. Volume 31, Issue 5 (special issue: Summarizing Text) Pages: 735 - 751.September 1995.

3. Schreiber G., Akkermans H., Anjewierden A., De Hoog R., Shadbolt N., Van de Velde W., Wielinga B.: "Knowledge engineering and management. The CommonKADS methodology" MIT Press, 2000.

4. Molina M., Flores V.: "A knowledge-based approach for automatic generation of summaries of behavior”. Proc. 12th International Conference on Artificial Intelligence: Methodology, Systems, Applications. AIMSA 06. Lecture Notes of Artificial Intelligence. Springer. Varna, Bulgaria, 2006.

5. Iwasaki Y. and Low C.: "Model Generation and Simulation of Device Behavior with Continuous and Discrete Changes”. Intelligent Systems Engineering, Vol. 1 No.2. 1993.

6. Gruber T. R. and Olsen G. R.: “An Ontology for Engineering Mathematics”. In Jon Doyle, Piero Torasso, \& Erik Sandewall, Eds., Fourth International Conference on Principles of Knowledge Representation and Reasoning, Gustav Stresemann Institut, Bonn, Germany, Morgan Kaufmann, 1994.

7. Borst P., Akkermans J. M., Pos A., and Top. J. L.: “The PhysSys ontology for physical systems”. In R. Bredeweg, editor, Working Papers Ninth International Workshop on Qualitative Reaso ning QR'95. Amsterdam, NL, May 16-19. 1995.

8. Mann W.C., Thompson S.A.: "Rhetorical Structure Theory: Toward a Functional Theory of Text Organization”. Text journal, Vol. 8, N. 3, 243-281. 1988.

9. Ghallab M., Nau D., Traverso P.: “Automated Planning: Theory and Practice”. Morgan Kaufmann, 2004.

10. Molina, M.: "Building a Decision Support System with a Knowledge Modeling Tool". Journal of Decision Systems. Special issue "Design, Building and Evaluation of Intelligent Decision-making Support Systems". Lavoisier, France, 2006.

11. Benjamins R.: "Problem-solving methods for diagnosis". PhD thesis, University of Amsterdam, Amsterdam, The Netherlands. 1993.

12. Wahlster W., André E., Graf W., Rist, T.: "Designing illustrated texts: how language production is influenced by graphics generation", Proceedings of the fifth conference on European chapter of the Association for Computational Linguistics, p.8-14, April 09-11, 1991, Berlin, Germany.

13. Reiter E.: “NLG vs. Templates”. In Fifth European Workshop on Natural Language Generation”. Leiden, 1995.

14. O'Donnell M.: "Intermixing Multiple Discourse Strategies for Automatic Text Composition". Revista Canaria de Estudios Ingleses (RCEI), No. 40 (April): Special Issue on Intercultural and Textual Approaches to Systemic-Functional Linguistics, 2000.

15. Kerpedjiev S. M.: "Automatic generation of multimodal weather reports from datasets". Proceedings of the third Conference on Applied Natural Language Processing. Trento, Italy. $48-55.1992$.

16. Kittredge R., Polguere A., Goldberg E.: "Synthesizing weather forecasts from formatted data”. COLING-86, Proceedings, Bonn, August 1986.

17. Bourbeau L., Carcagno D., Goldberg E., Kittredge R., Polguere A.: "Synthesizing Weather Forecasts in an Operational Environment”. In Proc. $13^{\text {th }}$ International Conference COLING, vol. 3, 318-320, Helsinki, August 1990. 